\title{
Should we marry a pharmacist? With or without separate property
}

\author{
Elisa Worthington*, Mette Juul-Gregersen \\ From 6th Danish Emergency Medicine Conference \\ Odense, Denmark. 20-21 November 2014
}

\section{Background}

In health care, there are increasing demands for efficiency and safe patient care. Concomitantly, requirements to patient-related documentation have risen among others for medication. Documentation is, however, time consuming and therefore not always done diligently, perhaps especially on weekends. One proven way to improve patient safety is the review of medication by a pharmacist after admission to the hospital to ensure correct medication (and documentation). Should we accept that pharmacists perform a medication review for us?

\section{Material and methods}

From May 1 to May 31, 2014, we did a non-randomized retrospective study of 259 consecutive surgical patients admitted to the Emergency \& Trauma Center, AAUH. The focus was on medication review (MG), aligned the medication (MA), medication status (MS) and registration of medical allergies (CAVE registration) at admission and at discharge.

\section{Results}

At admission MG was made in $41.7 \%$ of the cases, MA of $40.5 \%$, MS $75.7 \%$, and in $17 \%$ of patients no medication review was performed. On discharge MG was made in $24.3 \%$ of the cases, MA $36.3 \%$, MS $34.7 \%$, and in $43.6 \%$ no review. In a total of 24 patients $(9.3 \%)$, no review whatsoever of medicine was performed, neither in the ED nor throughout the in-hospital stay. Twelve cases (50\%) occurred between Friday and Sunday. CAVE registration did not take place in 37 patients (14.3\%). Of these $48.6 \%$ (18 cases), happened in the weekend.

* Correspondence: anew@rn.dk

Akut og Traumecenter, Ålborg Universitetshospital (AAUH), Aalborg Denmark 